\title{
Potential of Cellulose-Based Superabsorbent Hydrogels as Water Reservoir in Agriculture
}

\author{
C. Demitri, F. Scalera, M. Madaghiele, A. Sannino, and A. Maffezzoli \\ Department of Engineering for Innovation, University of Salento, Via per Monteroni, 73100 Lecce, Italy \\ Correspondence should be addressed to C. Demitri; christian.demitri@unisalento.it
}

Received 19 July 2013; Revised 26 November 2013; Accepted 6 December 2013

Academic Editor: Aihua He

Copyright (C) 2013 C. Demitri et al. This is an open access article distributed under the Creative Commons Attribution License, which permits unrestricted use, distribution, and reproduction in any medium, provided the original work is properly cited.

\begin{abstract}
The present work deals with the development of a biodegradable superabsorbent hydrogel, based on cellulose derivatives, for the optimization of water resources in agriculture, horticulture and, more in general, for instilling a wiser and savvier approach to water consumption. The sorption capability of the proposed hydrogel was firstly assessed, with specific regard to two variables that might play a key role in the soil environment, that is, ionic strength and $\mathrm{pH}$. Moreover, a preliminary evaluation of the hydrogel potential as water reservoir in agriculture was performed by using the hydrogel in experimental greenhouses, for the cultivation of tomatoes. The soil-water retention curve, in the presence of different hydrogel amounts, was also analysed. The preliminary results showed that the material allowed an efficient storage and sustained release of water to the soil and the plant roots. Although further investigations should be performed to completely characterize the interaction between the hydrogel and the soil, such findings suggest that the envisaged use of the hydrogel on a large scale might have a revolutionary impact on the optimization of water resources management in agriculture.
\end{abstract}

\section{Introduction}

Superabsorbent hydrogels are a particular class of macromolecular gels, obtained by chemical stabilization of hydrophilic polymers in a three-dimensional network, in which the dispersed phase is water, present in substantial quantity. Currently, superabsorbent hydrogels are widely used as absorbent core for hygiene products (such as baby diapers), and this attractive business has motivated the interest of multinational companies toward the development of new technologies, with focus on both the "chemical definition" and the production processes of these materials [1-5].

However, most of the superabsorbents that are currently on the market are acrylate-based products; hence, they are not biodegradable and, most importantly, some concerns exist about their toxicity for use in agriculture or for any applications related to human consumption. As a result, the renewed attention of institutions and public opinion towards the Environment has led manufacturers of hydrogel-based products to consider the development of biodegradable superabsorbents [6-13].
Sannino and coworkers developed and patented a novel class of cellulose-based polyelectrolyte hydrogels, totally biodegradable and biocompatible, whose swelling capability can be modulated by adjusting several synthesis parameters [14-20]. Such hydrogels may thus absorb up to 1 litre of water or aqueous solutions per gram of dry material. The material can be produced in the dry state either in powder form or as a bulk with a well-defined shape (in this regard, it is worth noting that the material displays a strong memory of its shape after swelling). Additionally, the hydrogel can be loaded with small molecules, for example, nutrients, to be released in a controlled manner, depending on swelling-deswelling transitions [13].

The aim of this study was to investigate the potential of cellulose-based hydrogels, crosslinked by means of a watersoluble carbodiimide, as a system for the sustained release of water (and nutrients) to plant roots, for agriculture and horticulture in arid and desert areas. The biocompatibility of this class of hydrogels had been previously confirmed [17, 18] and suggested their possible application in agriculture. The hydrogel is envisaged to be produced in form of powder, 


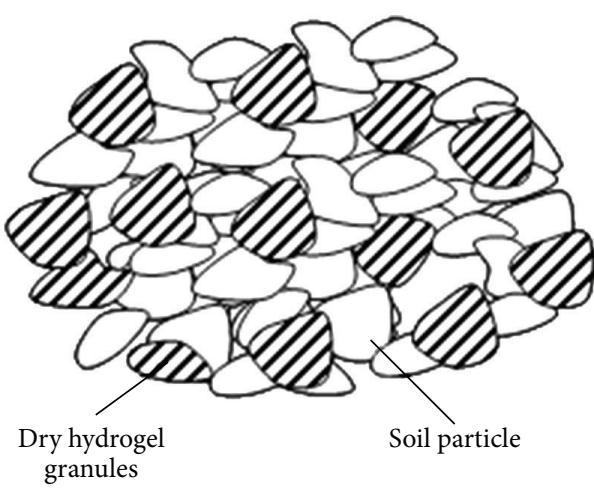

(a)

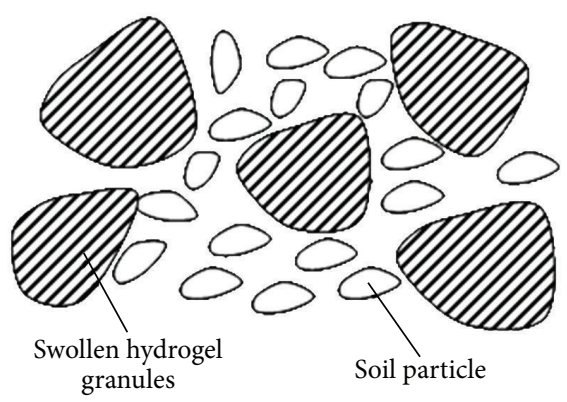

(b)

FIGURE 1: Effect of hydrogel swelling on soil porosity: (a) dry hydrogel, (b) swollen hydrogel. Adapted from [13].

possibly charged with nutrients and/or plants pharmaceuticals, and mixed to the soil in proximity of the plant roots. When the cultivation is watered (by either irrigation or rain), the hydrogel absorbs and retains the water, thus avoiding a rapid water loss due to evaporation and drainage. As the soil dries, the hydrogel releases the stored water in a sustained manner through a diffusion mechanism, keeping the soil or the substrate humid over quite long periods of time. A further advantage in mixing the hydrogel with the soil is that hydrogel granules (which in the dry form have almost the same dimensions of the substrate granules) increase their size after swelling, thus increasing soil porosity and providing a better oxygenation to the plant roots, as schematized in Figure 1.

In this study, polyelectrolyte cellulose-based hydrogels were synthesized and tested for swelling capability in different environmental conditions (i.e., different values of ionic strength and $\mathrm{pH}$ of the external solution), which might significantly affect the hydrogel swelling and deswelling occurring in the soil. The application of the hydrogel for the cultivation of "cherry tomatoes," typical of Southern Italy, was also investigated in a pilot study.

\section{Materials and Methods}

2.1. Hydrogel Synthesis. Carboxymethylcellulose sodium salt $(\mathrm{CMCNa})$ and hydroxyethylcellulose (HEC) were used as precursors for the hydrogel synthesis. In particular, $\mathrm{CMCNa}$ (Blanose 7HOF, with MW $7 \times 10^{5} \mathrm{Da}$, DS $=0.7$, viscosity $1000-2800 \mathrm{cp}\left(1 \%, 25^{\circ} \mathrm{C}\right)$ ) and HEC (Natrosol $250 \mathrm{HR}$, with MW $10^{6} \mathrm{Da}, \mathrm{MS}=2.5$, viscosity $1500-2500 \mathrm{cp}$ $\left.\left(1 \%, 25^{\circ} \mathrm{C}\right)\right)$, both for food and pharmaceutical applications, were purchased from Eigenmann and Veronelli, Milano, Italy. The crosslinking agent used was 1-ethyl-3-(3dimethylaminopropyl)carbodiimide hydrochloride (WSC) (Sigma-Aldrich, Milano, Italy). Three different hydrogel formulations were synthesized (Table 1), by varying the total cellulose concentration in the starting polymer solution $(3,4$, and $5 \mathrm{wt} \%)$. The amount of the crosslinking agent (2.5 wt\%) and the $\mathrm{CMCNa} / \mathrm{HEC}$ weight ratio (3/1) were kept constant, based on the results obtained in previous works [14-20].
A mixture of $\mathrm{CMCNa}$ and HEC was first dissolved in distilled water by stirring at room temperature until a clear solution was obtained. First, HEC was easily dispersed, after which $\mathrm{CMCNa}$ was added. $\mathrm{CMCNa}$ dissolution was slow at the concentration adopted, requiring about 24 hours. Once the cellulose solution was obtained, an aqueous solution of citric acid ( $1 \% \mathrm{w} / \mathrm{v})$ was added as a catalyst. The carbodiimide was finally added and allowed to dissolve and distribute homogeneously throughout the solution. The crosslinking reaction occurred in a few hours at room temperature, leading to the formation of a yellowish hydrogel. To remove the unreacted chemicals, the partially swollen hydrogel samples were then soaked in a large amount of distilled water until equilibrium was attained. At this stage, the hydrogel samples were perfectly clear and transparent. After the washing stage, dry products were obtained by desiccating the hydrogel samples by means of a thermal treatment in a convection oven at $45^{\circ} \mathrm{C}$. Powder-like samples were finally obtained by grinding. The final powder size distribution was in the range $0.1-1 \mathrm{~mm}$.

2.2. Swelling Measurements. The hydrogel swelling capability was firstly assessed in distilled water. Both equilibrium and kinetic measurements were performed, as the water holding capacity and the sorption rate of the hydrogel are both important for the envisaged application in agriculture. The equilibrium swelling capability was measured by carefully weighing the samples before and after immersion in distilled water for about 24 hours. A tea bag was employed to easily manipulate hydrogel samples after swelling. The swelling ratio (SR) was defined as follows:

$$
\mathrm{SR}=\frac{W_{s}-W_{d}}{W_{d}}
$$

where $W_{s}$ is the weight of the swollen hydrogel and $W_{d}$ is the weight of the dried sample.

The hydrogel sorption rate was then assessed by monitoring the weight gain of the samples after their immersion in distilled water for different time lengths $(5,10,15,20,25,30$, 60,120 , and 180 minutes) and expressed as SR variations. 
TABLE 1: Hydrogel formulations tested, differing for cellulose concentration.

\begin{tabular}{lc}
\hline Sample ID & $\begin{array}{c}\text { Cellulose concentration } \\
\% \mathrm{w} / \mathrm{v}\end{array}$ \\
\hline $\mathrm{A}$ & $3 \%$ \\
$\mathrm{~B}$ & $4 \%$ \\
$\mathrm{C}$ & $5 \%$ \\
\hline
\end{tabular}

Equilibrium swelling studies at different ionic strength were also performed, by preparing four water solutions of $\mathrm{NaCl}$ at concentrations of $10^{-3}, 10^{-2}, 10^{-1}$, and $1 \mathrm{M}$. The dry samples were weighed and immersed in the aqueous solution until equilibrium attainment. The same procedure was adopted for the analysis of the swelling capacity at different $\mathrm{pH}$ (in the range of $\mathrm{pH}$ values 2-10). In this case, a proper amount of $\mathrm{NaCl}$ was added to the buffer solutions used, in order to obtain the same value of ionic strength $(0.04 \mathrm{M})$.

All of the swelling measurements, both in distilled water and in water solutions, were carried out at room temperature using an electronic microbalance (Sartorius) with an accuracy of $\pm 10^{-5} \mathrm{~g}$. Reported values of water uptakes for each different hydrogel composition were averaged over five measurements.

2.3. Pilot Study on the Application of the Hydrogel in Agriculture. With the aim of further exploring the possible application of the hydrogel as water reservoir in agriculture and horticulture, its direct effect on the cultivation of a "cherry tomato," specialty from the south of Italy was assessed in experimental greenhouses. Dry hydrogel powder of type "B," which had been selected based on the results obtained from the swelling analyses, was mixed to the soil (red soil) in the area closed to the plant roots. Different amounts of the hydrogel were added to the soil, up to concentrations equal to $0.2 \%, 0.5 \%$, and $1 \%$ (wt $\%$ ) of the soil. The soil without the amendment of the hydrogel was used as a control. The soil was then watered and its humidity content (expressed as \% volume) was constantly monitored through a custom-made sensor, already described in the literature $[21,22]$, which exploits the hydrogel itself as a highly sensitive detection system. Such a sensor, able to measure the humidity conditions of the hydrogel in the soil, is particularly useful to determine the optimal time point at which it is necessary to irrigate the soil.

The effect of different hydrogel amounts $(0.5 \%, 1 \%$, and $2 \%$ ) on the soil water retention curve was also investigated, by means of a custom design pressure plate apparatus [23].

\section{Results and Discussion}

3.1. Swelling Measurements. The equilibrium swelling tests in distilled water showed that sample B ( $4 \mathrm{wt} \%$ cellulose) displayed higher swelling ratios when compared with samples A (3 wt $\%$ cellulose) and C (5wt\% cellulose) (Figure 2). Cellulose concentration in the precursor solution was thus found to significantly affect the hydrogel sorption properties, with

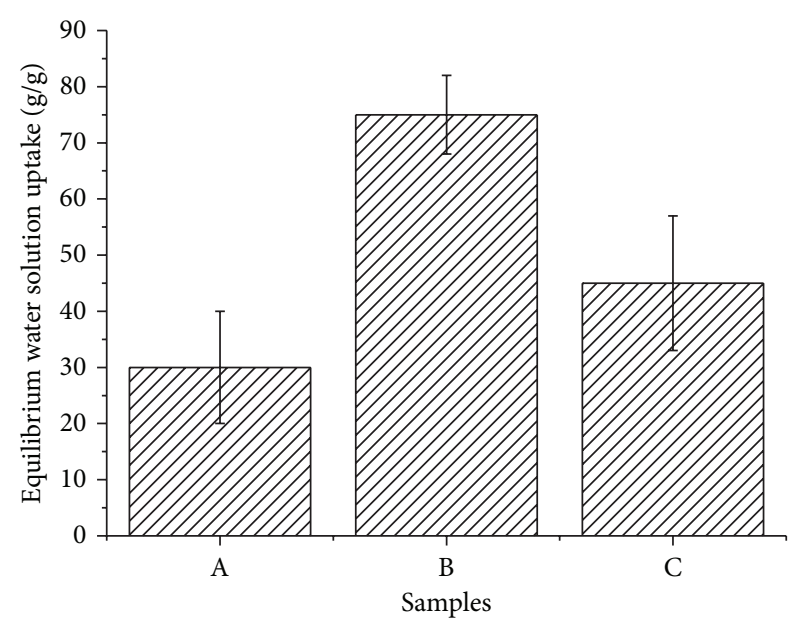

FIGURE 2: Hydrogel equilibrium swelling properties in distilled water. Results are reported as mean \pm standard deviation of the mean $(n=5)$.

the highest sorption capability attained at an intermediate value of cellulose concentration. This particular behaviour could be explained by considering the role played by two competitive mechanisms. On one side, high polymer concentrations in the starting solution (e.g., $5 \mathrm{wt} \%$ ) cause the formation of a high number of physical entanglements, which, after chemical crosslinking, act as further crosslinking points, thus reducing the hydrogel swelling capability. On the other, low polymer concentrations (e.g., $3 \mathrm{wt} \%$ ) reduce the efficacy of the crosslinking reaction, due to a low concentration of reactive sites in solution, which leads to loosely crosslinked networks with poor sorption capacity. This was likely why optimal swelling capability was displayed by sample B, which had been obtained from a precursor solution with an intermediate value of cellulose concentration. Hydrogel B was thus selected for further analyses.

The kinetic swelling measurements in distilled water showed a fast uptake for all the hydrogel formulations (Figure 3). After 30 minutes, all the samples almost reached the equilibrium values of swelling ratio. Indeed a stable "plateau" region could be observed in the time frame between 30 and 180 minutes. A slight increase of the swelling ratio could be detected for sample B only, although the swelling ratio attained at 30 minutes was very close to the average equilibrium value (Figure 2). A time length of 30 minutes was thus considered as a reasonable and acceptable duration for complete hydrogel swelling, when applying the hydrogel in agriculture.

The effect of the ionic strength of the external solution on the equilibrium sorption properties of the hydrogels is shown in Figure 4. As expected, all of the samples tested were found to be particularly sensitive to ionic strength variations, due to the presence of the polyelectrolyte $\mathrm{CMCNa}$ in the polymer network, which is known to lead to the establishment of "Donnan type" equilibrium between the gel and the surrounding solution. Obviously, for each sample, increasing the ionic strength of the external solution decreased the difference between the concentration of ion species in the gel 


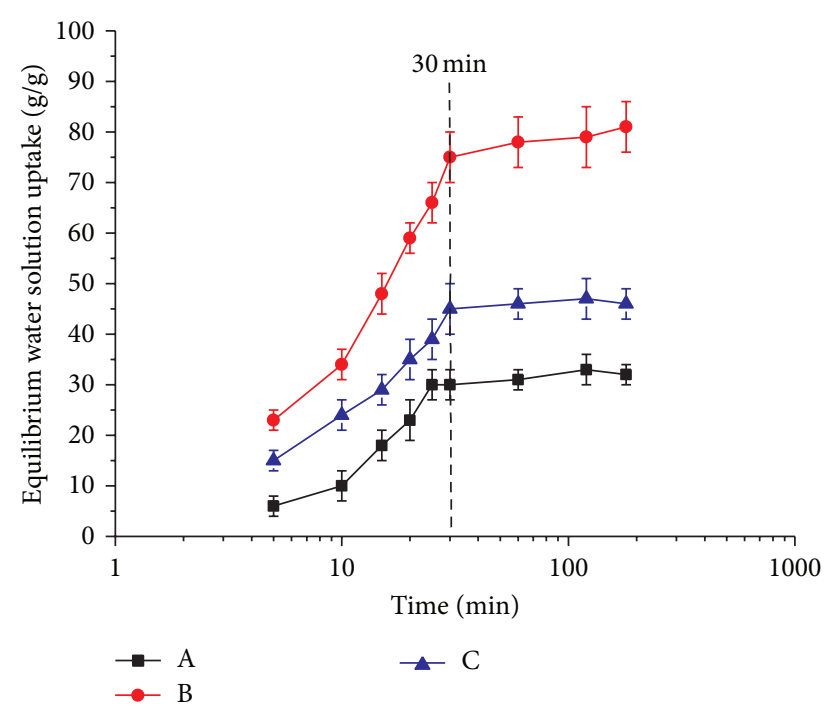

FIGURE 3: Hydrogel swelling kinetics in distilled water. Results are reported as mean \pm standard deviation of the mean $(n=5)$.

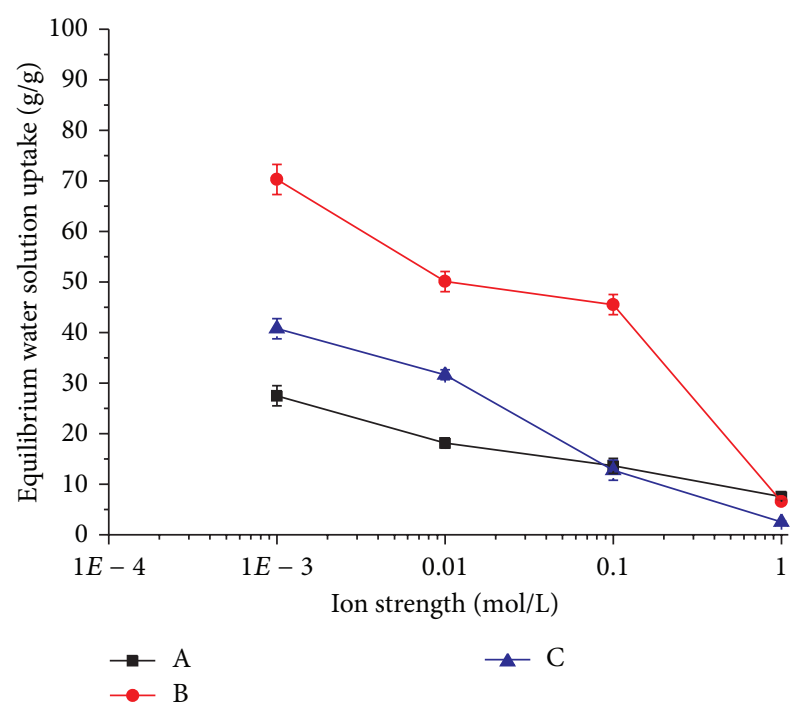

FIGURE 4: Hydrogels equilibrium swelling properties in water solution at different ionic strength. Reported values have been averaged over five measurements $(n=5)$.

and in the external solution and, as a result, the water uptake. The effect of ionic strength was particularly evident for sample B, which, however, displayed the highest swelling capability even at high ionic strength values (e.g., 0.1 M).

With regard to the hydrogel sensitivity to $\mathrm{pH}$ variations, an increase of the swelling ratio could be observed when increasing the $\mathrm{pH}$ of the external solution (Figure 5). This was related to the dissociation of the carboxylic groups present on CMCNa chains, which clearly depends upon the $\mathrm{pH}$ of the surrounding environment. As the $\mathrm{pH}$ of the external solution decreases, the number of negative charges tethered to the polymer backbone diminishes, since $\mathrm{H}^{+}$ions will be associated to the carboxylic groups brought by CMCNa. This reduces the ionic "Donnan type" contribution to the hydrogel

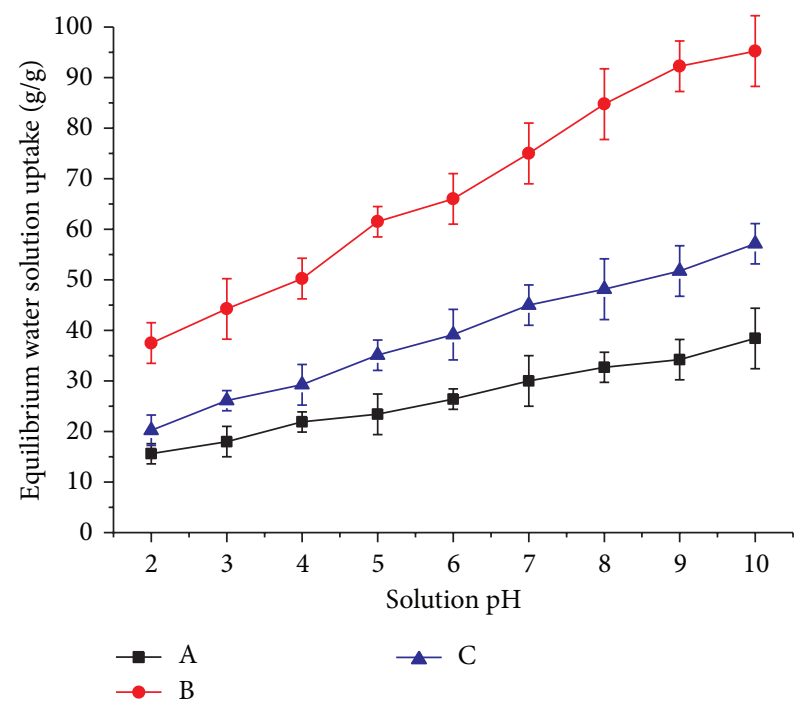

FIgURE 5: Plot of the equilibrium swelling ratio versus the $\mathrm{pH}$ of the external solution bathing the hydrogel $(n=5)$.

equilibrium swelling capacity, thus resulting in lower swelling ratios. The supply of $\mathrm{H}^{+}$ions from the dissociated groups on the backbone is, however, limited. This suggests that, at a certain high value of the $\mathrm{pH}$, all the carboxylic groups will be dissociated, giving rise to a fully charged network and thus leading to a maximum value of swelling capability.

3.2. Pilot Study and Preliminary Observations. The use of the hydrogel as water reservoir in the cultivation of a "cherry tomato" (i.e. a small rounded tomato) in experimental greenhouses (Figure 6) was found to be extremely advantageous for the sustained release of water to the soil and to the plant roots.

The water content in the soil, monitored over several days after initial irrigation, was indeed significantly affected by the presence of the hydrogel, with higher water contents detected at selected time points, when increasing the hydrogel concentration (Figure 7).

It is particularly interesting to note that, after the addition of small hydrogel amounts (up to a maximum of 1\%), the time length through which the soil remained humid almost triplicated, with respect to the soil devoid of hydrogel. Moreover, the initial humidity, right after watering, was substantially higher for the soil where the hydrogel had been added, thus confirming its water reservoir or storage capability. Indeed, the relatively fast swelling of the hydrogel powder allows the irrigated water to be efficiently retained in the soil, instead of being naturally lost by means of evaporation and/or drainage. It is worth mentioning that the evaporation of water depends upon the climate conditions. However, the evaporation from a macromolecular hydrogel is significantly slower than the evaporation of free water from a soil exposed to atmosphere, due to the macromolecular network hindrance and the interaction between water and polymer molecules. As the humidity content in the surrounding soil decreases, the absorbed water is then slowly released by the hydrogel, through a diffusion driven mechanism. 


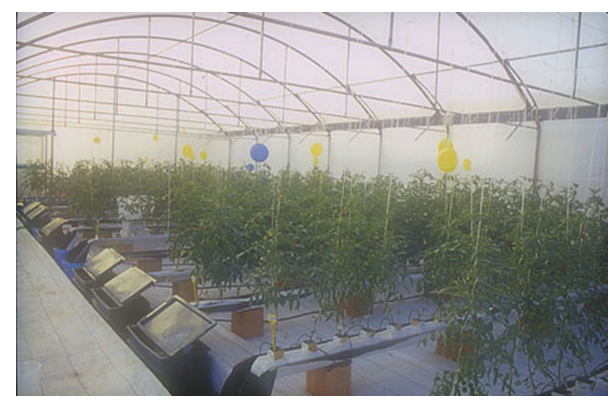

(a)

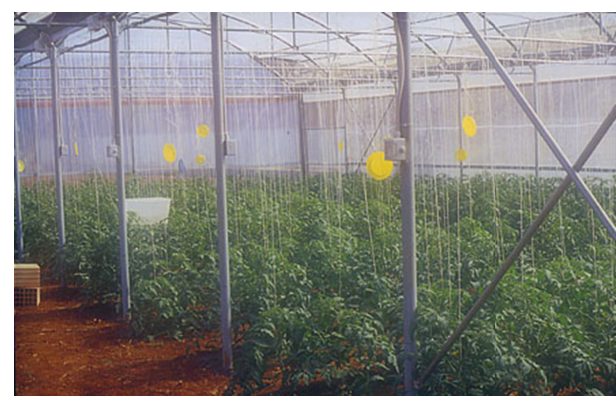

(b)

FIGURE 6: The greenhouses in the south of Italy where the Hydrogel effect on the cultivation of tomatoes was preliminarily tested.

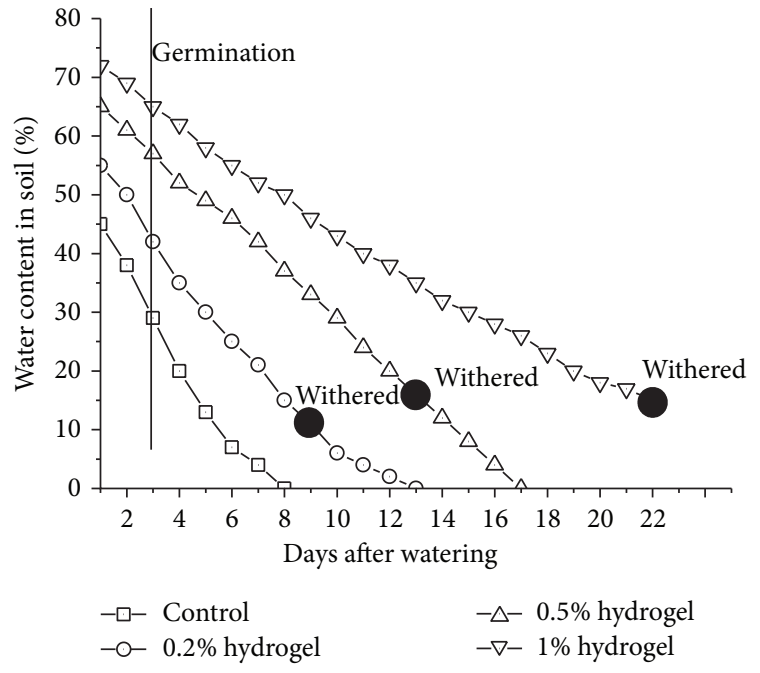

FIGURE 7: Water content in the soil as a function of the number of days after watering: effect of different hydrogel contents.

The controlled and sustained release of water allows plants to survive for several days without the need of further irrigations. In particular, the tomato plants cultivated in this study were found to wither when the soil humidity reached values in the range $10-20 \%$ (or lower). Such values were attained in about 9 days when only $0.2 \%$ of hydrogel was added to the soil. Conversely, a much longer plant survival time, equal to 22 days, was achieved when $1 \%$ hydrogel was used (Figure 7). In the long run, if envisaging an efficient and large scale use of the proposed hydrogel in agriculture, the hydrogel-based sensor developed to monitor that the soil humidity might be integrated also with a specific computer software, able to activate an automatic irrigation system as the humidity content in the hydrogel (and soil) reaches a critical low level.

In order to confirm the water reservoir role of the hydrogel suggested by the above reported results, additional measurements of the suction potential of the soil were performed. As expected, the hydrogel-amended soil displayed a higher water retention capability compared to the hydrogelfree control, and the water storage was significantly increased as higher hydrogel amounts were used (Figure 8).

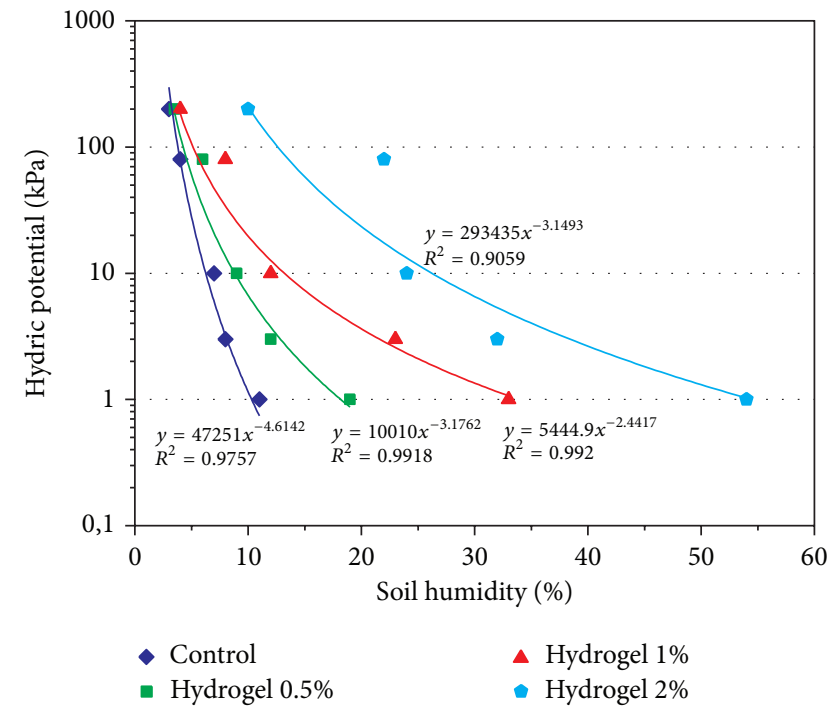

FIGURE 8: Effect of hydrogel concentration on soil suction potential: both saturated and residual water content increase as hydrogel concentration is increased.

Although further investigations should be performed to assess the hydrogel degradation chemistry and kinetics in the soil, as well as to evaluate the optimal hydrogel amount to be used for the cultivation of different plant species, the preliminary results of this pilot study show promise for the possible use of cellulose-based hydrogels, crosslinked by means of carbodiimide, as water reservoir in agriculture. With regard to the hydrogel degradation, analyses that are currently in progress seem to suggest that the hydrogel degradation occurs over a period of approximately 6 months, without significantly altering the soil chemistry.

\section{Conclusions}

In this work, polyelectrolyte cellulose-based hydrogels obtained from cellulose derivatives of pharmaceutical grade were investigated as potential water reservoir in agriculture. Several formulations were prepared, differing for the cellulose concentration in the precursor solution, using a watersoluble carbodiimide as a nontoxic crosslinker. The swelling 
capability was tested in different environmental conditions, that is, ionic strength and $\mathrm{pH}$, which might significantly affect the envisaged application in the soil. Based on the swelling data, a specific hydrogel formulation (the one obtained for a cellulose concentration equal to $4 \%$ ) was chosen for further analyses, as it displayed the highest water holding capacity. Being a polyelectrolyte, the selected hydrogel was also sensitive to ionic strength and $\mathrm{pH}$ variations, though the swelling capability was quite high in all the different conditions tested. A pilot study focused on the cultivation of tomatoes with hydrogel-amended soil demonstrated that the hydrogel could significantly increase the water retention capability of the soil, as well as allowing the sustained release of water to the plants for a prolonged time, without additional watering needed. A deeper analysis of the hydrogel degradation and its effect on the soil properties should be addressed in future investigations. However, the preliminary results of this study suggest that the proposed hydrogel might be a powerful means for optimizing water management in agriculture, which is particularly critical in areas where water scarcity is a severe problem.

\section{Acknowledgments}

The authors wish to acknowledge Dr. Francesco Serio from the Institute of Sciences of Food Production of the National Research Council ISPA-CNR (Italy) and Dr. Pietro Santamaria from the University of Bari (Italy) for their helpful support in performing the pilot study on hydrogel-amended soil and its effect on the cultivation of tomatoes in experimental greenhouses. This investigation was partially funded by the COL.GEL grant project, from the Italian Ministry of Education, University and Research (MIUR).

\section{References}

[1] F. L. Buchholz, "Superabsorbent polymers: science and technology," in Proceedings of the ACS Symposium Series 573, F. L. Buchholz and N. A. Peppas, Eds., pp. 27-38, American Chemical Society, Washington, DC, USA, 1994.

[2] T. Sakiyama, C.-H. Chu, T. Fujii, and T. Yano, "Preparation of a polyelectrolyte complex gel from chitosan and K-carrageenan and its pH-sensitive swelling," Journal of Applied Polymer Science, vol. 50, no. 11, pp. 2021-2025, 1993.

[3] M. Yoshida, M. Asano, and M. Kumakura, "A new temperaturesensitive hydrogel with $\alpha$-amino acid group as side chain of polymer," European Polymer Journal, vol. 25, no. 12, pp. 1197$1202,1989$.

[4] T. Shiga, Y. Hirose, A. Okada, and T. Kurauchi, "Bending of ionic polymer gel caused by swelling under sinusoidally varying electric fields," Journal of Applied Polymer Science, vol. 47, no. 1, pp. 113-119, 1993.

[5] K. Hogari and F. Ashiya, Advances in Superabsorbent Polymers, American Chemical Society, Washington, DC, USA, 1994.

[6] M. Ben-Hur and R. Keren, "Polymer effects on water infiltration and soil aggregation," Soil Science Society of America Journal, vol. 61, no. 2, pp. 565-570, 1997.

[7] J. Levin, M. Ben-Hur, M. Gal, and G. J. Levy, "Rain energy and soil amendments effects on infiltration and erosion of three different soil types," Australian Journal of Soil Research, vol. 29, no. 3, pp. 455-465, 1991.

[8] A. R. Al-Harbi, A. M. Al-Omran, A. A. Shalaby, and M. I. Choudhary, "Efficacy of a hydrophilic polymer declines with time in greenhouse experiments," HortScience, vol. 34, no. 2, pp. 223-224, 1999.

[9] M. P. Raju and K. M. Raju, "Design and synthesis of superabsorbent polymers," Journal of Applied Polymer Science, vol. 80, no. 14, pp. 2635-2639, 2001.

[10] L. Xie, M. Liu, B. Ni, and Y. Wang, "Utilization of wheat straw for the preparation of coated controlled-release fertilizer with the function of water retention," Journal of Agricultural and Food Chemistry, vol. 60, no. 28, pp. 6921-6928, 2012.

[11] L. Wu and M. Liu, "Preparation and characterization of cellulose acetate-coated compound fertilizer with controlled-release and water-retention," Polymers for Advanced Technologies, vol. 19, no. 7, pp. 785-792, 2008.

[12] L. Wu, M. Liu, and R. L. Rui Liang, "Preparation and properties of a double-coated slow-release NPK compound fertilizer with superabsorbent and water-retention," Bioresource Technology, vol. 99, no. 3, pp. 547-554, 2008.

[13] A. Sannino, C. Demitri, and M. Madaghiele, "Biodegradable cellulose-based hydrogels: design and applications," Materials, vol. 2, pp. 353-373, 2009.

[14] F. Esposito, M. A. Del Nobile, G. Mensitieri, and L. Nicolais, "Water sorption in cellulose based hydrogels," Journal of Applied Polymer Science, vol. 60, no. 13, pp. 2403-2407, 1996.

[15] A. Sannino and L. Nicolais, "Concurrent effect of microporosity and chemical structure on the equilibrium sorption properties of cellulose-based hydrogels," Polymer, vol. 46, no. 13, pp. 46764685, 2005.

[16] C. Demitri, F. Marotta, A. Sannino, E. S. Ron, Y. Zohar, and L. Ambrosio, "Rheological and mechanical comparison between natural dietary fibers and a novel superabsorbent biodegradable hydrogel (SAEF)," in Proceedings of the 24th European Conference on Biomaterials (ESB '11), Dublin, Ireland, 2011.

[17] A. Sannino, M. Madaghiele, C. Demitri et al., "Development and characterization of cellulose-based hydrogels for use as dietary bulking agents," Journal of Applied Polymer Science, vol. 115, no. 3, pp. 1438-1444, 2010.

[18] M. G. Raucci, M. A. Alvarez-Perez, C. Demitri, A. Sannino, and L. Ambrosio, "Proliferation and osteoblastic differentiation of hMSCS on cellulose-based hydrogels," Journal of Applied Biomaterials \& Functional Materials, vol. 10, no. 3, pp. 302-307, 2012.

[19] C. Demitri, R. Del Sole, F. Scalera et al., "Novel superabsorbent cellulose-based hydrogels crosslinked with citric acid," Journal of Applied Polymer Science, vol. 110, no. 4, pp. 2453-2460, 2008.

[20] A. Sannino, S. Pappadà, M. Madaghiele, A. Maffezzoli, L. Ambrosio, and L. Nicolais, "Crosslinking of cellulose derivatives and hyaluronic acid with water-soluble carbodiimide," Polymer, vol. 46, no. 25, pp. 11206-11212, 2005.

[21] A. Cataldo and E. De Benedetto, "Broadband reflectometry for diagnostics and monitoring applications," IEEE Sensors Journal, vol. 11, pp. 451-459, 2011.

[22] A. Cataldo, G. Monti, E. De Benedetto, G. Cannazza, and L. Tarricone, "A noninvasive resonance-based method for moisture content evaluation through microstrip antennas," IEEE Transactions on Instrumentation and Measurement, vol. 58, no. 5, pp. 1420-1426, 2009.

[23] E. Schlichting, H. P. Blume, and K. Stahr, Bodenkundliches Praktikum, Berlin, Germany, 1995. 

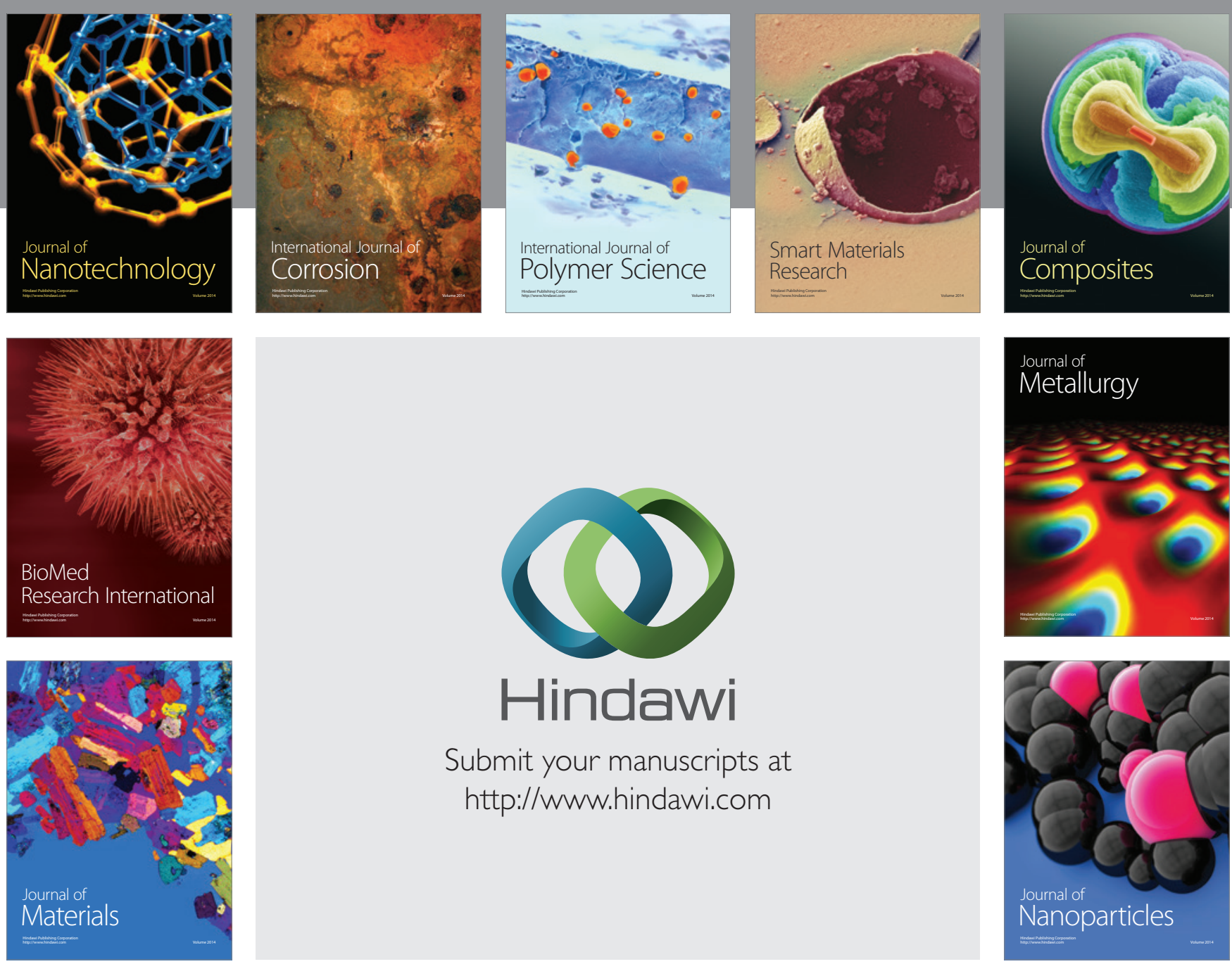

Submit your manuscripts at http://www.hindawi.com
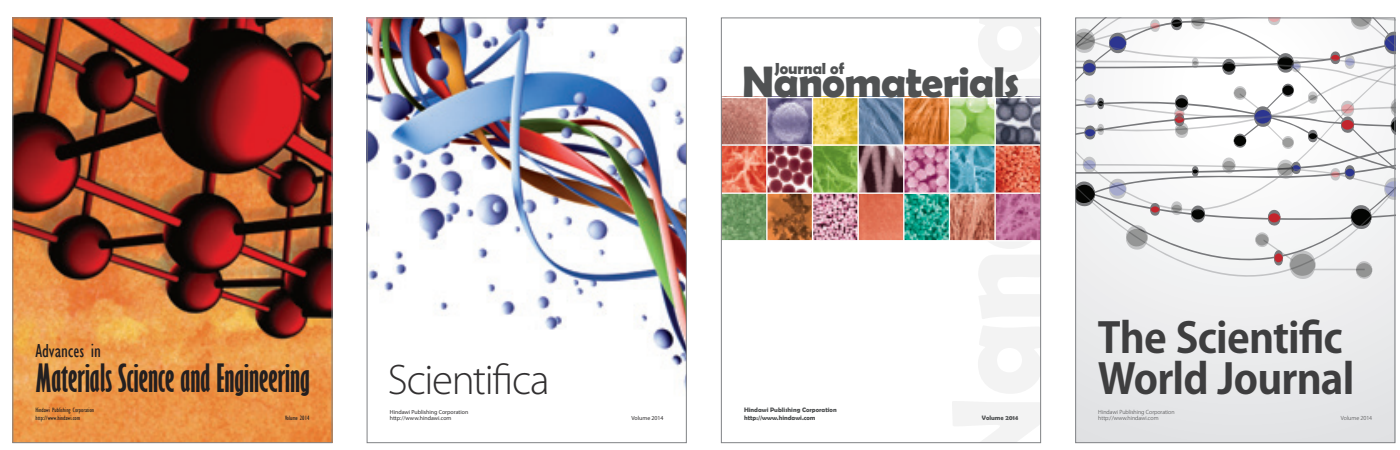

\section{The Scientific World Journal}
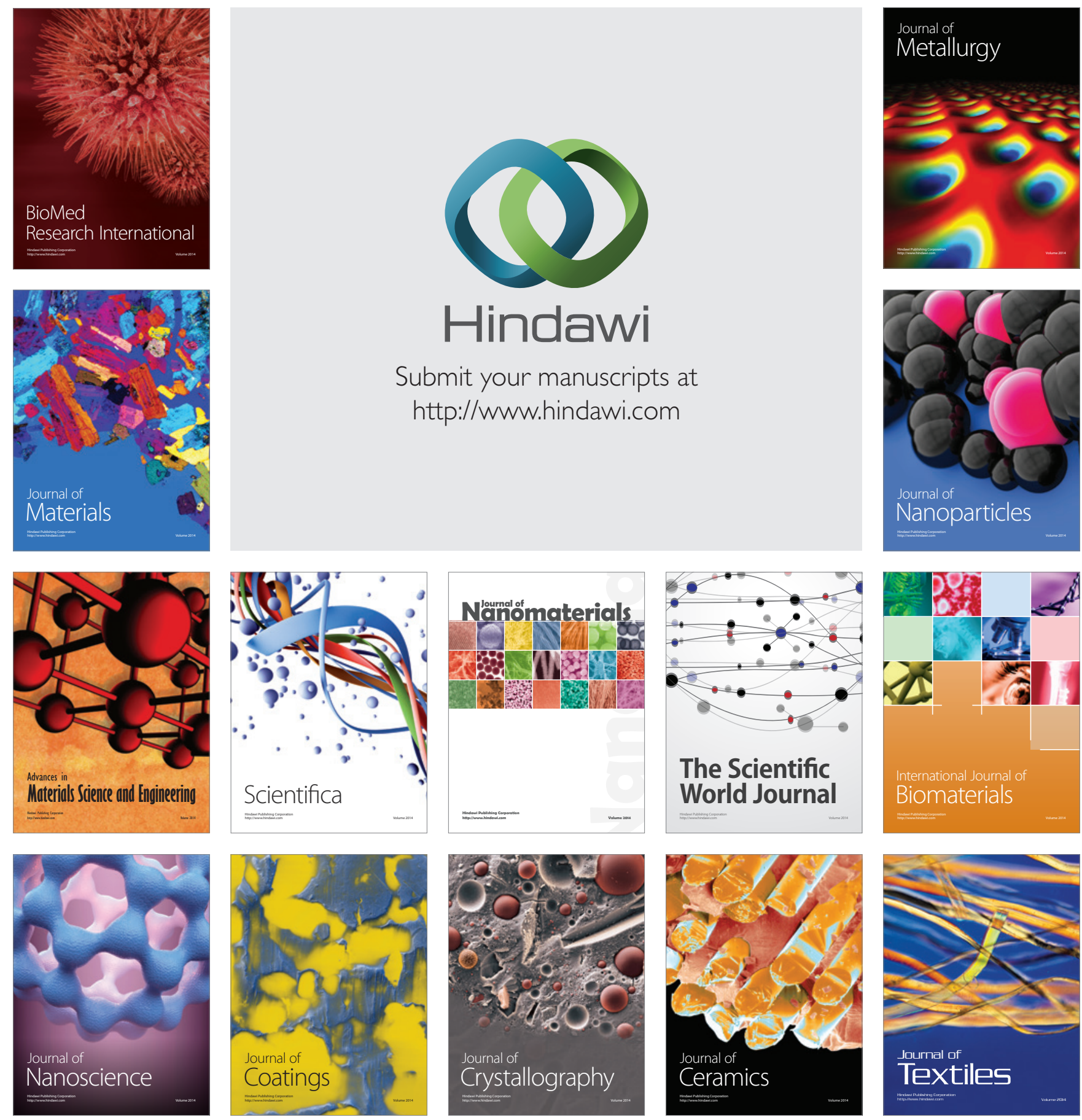